\title{
COLLAPSE OF THE LUNG ASSOCIATED WITH PRIMARY TUBERCULOUS LESIONS
}

\author{
BY

\section{A. MARGARET MACPHERSON AND V. URSULA LUTWYCHE From the Children's Contact Clinic, Brompton Hospital, London*}

With the increase in radiographic investigations in children and the increased possibilities for surgical intervention in tuberculous lesions of the thorax, the treatment of collapse of the lung associated with tuberculous hilar adenitis is a problem often raised. Questions arise as to whether or not any attempt should be made to free the airway to the collapsed lobe, whether the risk in so doing is greater than the risk of development of bronchiectasis later on, and at what stage hope of re-expansion of the collapsed lung should be abandoned.

We thought it might be worth recording some of the details, and especially the subsequent histories, of cases of collapse of the lung associated with active tuberculous lesions in the hilar glands found in children attending the Children's Contact Clinic at the Brompton Hospital during the last 17 years. The group is to a certain extent a selected one consisting of children attending for routine examination as contacts, and also children referred from other out-patient departments of the hospital or from other hospitals where they have been brought because of symptoms. This latter group, not necessarily contact children, but definitely ailing children, may give a falsely increased incidence of collapse of the lung in our series. Since the clinic is attached to a chest hospital and not to a children's hospital, there is a relatively greater number of older children than infants, a fact which may also influence the incidence rate, but in the opposite direction.

Since 1932, 2,042 new cases have attended the clinic, the majority, 1,457, being contact cases but the remaining 585 were not known to have been in contact with an open case of tuberculosis. Out of this total, 821 had positive tuberculin tests, and it is among these that the cases of collapse of the lung were found. There were 39 , that is $4.7 \%$, which showed definite collapse of one or more lobes or part of a lobe which could be recognized in an ordinary radiograph and in which collapse was associated with an active primary lesion. We have excluded all those in whom collapse was doubtful and also those who, although they had obvious collapse or bronchiectasis, had no evidence of a recent primary lesion. The series therefore contains only those cases with definite collapse associated with enlarged hilar glands due to recent tuberculous infection.

It was found that the incidence of collapse was relatively higher in the youngest age group (Table I). This is not surprising, as hilar adenitis is more marked when primary infection takes place in infancy than when the first infection is in later childhood and, in the young child, the bronchial wall is more vulnerable and the lumen more easily occluded.

* This work was aided by a grant from the Sir Halley Stewart Research Fund. 
TABLE I

InCidence of Collapse in Different Age Groups

\begin{tabular}{c|c|c|c}
\hline Age (Years) & Total No. of Cases & Collapse & Per Cent \\
\hline $0-4$ & 154 & 14 & 9.9 \\
\hline $4-8$ & 272 & 16 & 5.9 \\
\hline $8-12$ & 238 & 6 & 2.9 \\
\hline 12 and over & 157 & 3 & 1.9 \\
\hline
\end{tabular}

\section{CONTACT HistoRY}

A history of contact with an open case of tuberculosis was given in 30 of the 39 cases and, in 29, the child was either still in contact or had been in contact with a tuberculous person within the last three years.

\section{SYMPTOMS}

Most of the children with collapse of the lung had symptoms. Only 10 of the 39 had no symptoms and were brought for examination as contacts, the pulmonary condition being unsuspected until seen in the radiograph. Of the other 29 who had symptoms, cough was the most frequent complaint, being present in 25 cases. A history of a pyrexial attack at the time the child was brought to hospital or of attacks within the last six months was given in 11 cases. Other symptoms were lassitude, anorexia, and failure to gain weight. One child had pain in the chest and was found to have, in addition to collapse, a pleural effusion, and two others were referred from other hospitals with pleural effusions. Although the children were not necessarily ill, it was considered that rest at this stage was essential, and 35 out of the 39 children were recommended for admission to hospital or sanatorium. The other four were sent to convalescent homes or remained at home resting.

\section{LOBES AFFECTED}

In three cases more than one lobe was affected, in one of these collapse of two lobes was coincident, and in the other two the lobes collapsed in sequence. The incidence of collapse in the various lobes is shown in Fig. 1. The right upper and

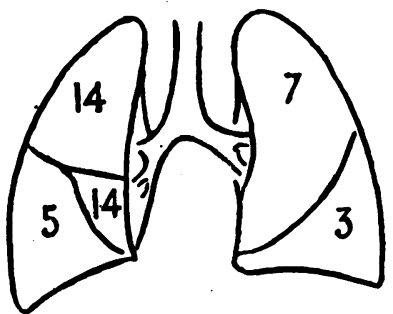

Fig. 1.-One lobe affected in 36 cases, two or more lobes affected in three cases. middle lobes were most frequently involved, then the left upper and right lower, and lastly the left lower lobe.

\section{SUBSEQUENT HISTORY}

In order to say with certainty whether or not the lung has re-expanded completely and whether or not there is residual bronchiectasis, it is essential to have a bronchogram. Unfortunately we were able to do bronchograms on only 13 of our cases. The period of observation included the war years when it was out of the question to admit children to a hospital in London for a bronchogram, and, once the child had 
returned to normal health and had remained well for two or three years, it was difficult to persuade the parents that a bronchogram was desirable. We have therefore had to accept a less accurate criterion than we should have liked and, in so doing, have no doubt overlooked cases of bronchiectasis and some lesser degrees of collapse. We have assumed that the lung has re-expanded if the ordinary radiograph showed a normal picture, apart from calcified lesions, and the collapsed lobe seen in previous films was apparently normal. This, together with the disappearance of symptoms and the normal appearance and development of the child, means that for practical purposes there are no evident residual effects from the original collapsed lung.

Looking at the material from this clinical point of view, it is interesting to find how few have any residual disability. In seven of the cases followed for less than two years, collapse was still evident. As this period is too short to be of value in assessing the results, these cases have been excluded from the following figures. Among the remaining 32 cases which were followed for periods from 2 to 17 years, 28 showed no evidence of persisting collapse. Three had collapse of the lung persisting after being under observation for periods of 6 to 13 years, and one had a lobectomy for bronchiectasis which developed in the collapsed lobe. In more than half the cases re-expansion had taken place in one year's time and in a further seven in two years' time (Table II). In three of the cases the lung re-expanded after an

TABLE II

ANAlysis of 32 Cases Followed Up for Two Years or More

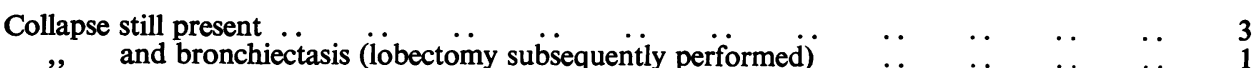

\begin{tabular}{lllllllllll} 
No evidence of collapse &. &. &. &. &. &. & $\ldots$ & $\ldots$ & $\ldots$ & \\
\hline
\end{tabular}

Total $\quad$. $\quad \ldots \quad \ldots \quad \overline{32}$

No collapse seen after an interval of:

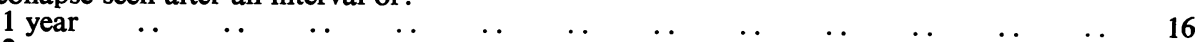

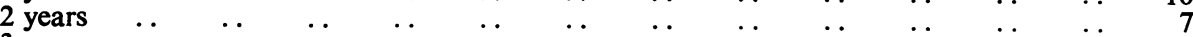

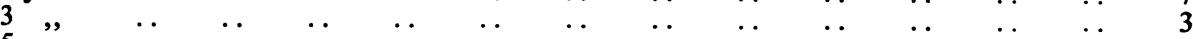

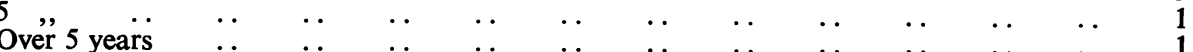

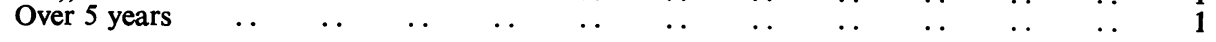

$\begin{array}{lllll}\text { Total } & \ldots & \ldots & \ldots & \overline{28}\end{array}$

interval of three years. The remaining two were not seen at regular intervals, and it was only possible to say that expansion had taken place within five years in one case and between 2 and 10 years in the other.

Of the four cases in which the lung did not re-expand, one had the left lower lobe removed for saccular bronchiectasis at the age of eight years, 18 months after she first came under observation. In the other three collapse has persisted. One has been under observation for 13 years and the other two for six years, and the patients are now aged 14, 16, and 17 years respectively. They are all normal, well-developed adolescents, and apart from the history from one of them of occasional coughs, they have no symptoms. The lobes affected in these cases are the right upper lobe in two cases and the right middle lobe in one. 


\section{BRONCHOGRAMS AND BRONCHOSCOPIES}

Bronchograms were done on 13 of the cases ; in five they confirmed the original diagnosis and in eight they confirmed either re-expansion (5) or bronchiectasis and persistent collapse (3).

Eight of the cases were bronchoscoped in the early stages when collapse was present. Changes in the lung following bronchoscopy were not striking; in half the cases there was no alteration in the degree of collapse ; in three there was some reexpansion within two months of bronchoscopy, and in one there was temporary partial re-expansion but shortly afterwards a recurrence of collapse.

\section{Discussion}

Although the numbers are small and the investigations are not as complete as we would have wished, this series of 32 cases of collapse of the lung resulting from primary tuberculous infection shows that permanent collapse and severe bronchiectasis are not common complications of primary tuberculosis. Out of the 821 children infected by the tubercle bacillus who came under observation only one has required surgical treatment for bronchiectasis, and among 32 cases of collapse of lung under observation for more than two years the lungs in all but three have apparently re-expanded. It is probable that with our limited means of investigation we have overlooked cases with lesser degrees of persisting collapse or bronchiectasis, but, if so, these are not giving rise to any clinical evidence during childhood or adolescence. This is an interesting result when one considers that, of the complications following primary tuberculous infection, collapse of lung resulting from hilar adenitis is one of the most frequent, and that tuberculous hilar adenitis in childhood is an important aetiological factor in bronchiectasis in later life. The tuberculous material in the lumen of the bronchus is only a small part of the much larger caseating tuberculous lesions in the glands and, as these latter may take two years or more to organize and heal, the bronchial lumen may remain occluded during this period, and one may reasonably continue to expect re-expansion up to or even beyond this length of time. In our cases in which the lung did not re-expand, we were unable to find any adequate reason for this failure to recover. It is possible that infection played a part, but there did not appear to be any infection worthy of note in these cases. In one of them, with atelectasis of the right upper lobe, subsequent bronchoscopy showed no constriction of the bronchi but only slight distortion near the orifice of the eparterial bronchus, so that permanent constriction in this case at least cannot be a contributory factor.

\section{SUMMARY}

Among children attending the Children's Contact Clinic at the Brompton Hospital during the last 17 years, there were 39 cases of collapse of a lobe or of lobes of the lung associated with primary tuberculous infection. These are considered with special reference to the incidence of the lobes affected, the incidence in varying age groups, and the symptoms associated with the condition. The subsequent histories of the cases over observation perinds up to 17 years are related. 